\title{
The integrated orderline batching, batch scheduling, and picker routing problem with multiple pickers: the benefits of splitting customer orders
}

\author{
Mustapha Haouassi ${ }^{1,2} \quad$ Jorge E. Mendoza $^{3} \quad$ Yannick Kergosien $^{1}$ \\ Louis-Martin Rousseau ${ }^{2}$ \\ 1 Université de Tours, LIFAT EA 6300, CNRS, ROOT ERL CNRS 7002 \\ 64 Avenue Jean Portalis 37200 TOURS \\ \{mustapha.haouassi, yannick.kergosien\}@etu.univ-tours.fr \\ 2 Ecole polytechnique de Montréal, CIRRELT \\ 2900 Boulevard Edouard-Montpetit H3T 1J4, Montréal (Québec), Canada \\ \{mustapha.haouassi, louis-martin.rousseau\}@polymtl.ca \\ 3 HEC Montréal \\ 3000 Chemin de la côte Sainte Catherine H3T 2A7, Montréal (Québec), Canada \\ jorge.mendoza@hec.ca
}

Mots-clés : Order picking, Splitting customer orders, Hard due-dates

\section{Introduction and problem statement}

Fast delivery is one of the most popular services in e-commerce retail. It consists in shipping the items ordered on-line in short times. Among all warehousing activities, order picking is considered as the most time-consuming and costly process. In "manual picker to part" warehouses, (human) order pickers performs "picking tours" starting from depot, walking through aisles to pick items from storage locations, and coming back to depot. Pickers are directed using picking lists where the orderlines to retrieve in a single tour and their respective storage locations are itemized. Optimizing the picking process in e-commerce warehouses requires combining a wide range of planning problems in integrated approaches [1]. In this study, we focus on the integration of 3 planning problems: regrouping the orders into pick lists (order batching), designing the tour that retrieves each pick list (picker routing), and assigning the picking lists to a set of pickers and scheduling the lists assigned to each picker (picker scheduling) in order to optimize the total picking time. To speed up the picking operations, e-commerce warehouses implement new logistical practices, such as spliting the customer orders (assigning the orderlines of an order to multiple pickers). this practice is generally prohibited in the picking literature because of the excessive packing efforts it may induce. Nonetheless, new sorting technologies and logistical techniques (automated conveyors, put walls for instance) have emerged in the e-commerce warehouses making the order accumulation and packing procedure more efficient and leading e-commerce warehouses (Amazon for instance) to implement the splitting of customer orders [2]. We want through this study to demonstrate the benefits of splitting the customer orders in ecommerce warehouses. We thus generalize the integrated order batching, batching routing, and picker scheduling problem of [1] by allowing the splitting of customer orders, develop a route first-schedule second (RFSS) heuristic to solve the problem, and compare the results of our heuristic with that of the Iterated Local Search (ILS) of [1] developed for the no-spliting version of the problem.

A formal definition of the problem is as follows. Let $\mathcal{O}$ be the set of customer orders. Each order $o \in \mathcal{O}$ consists of few orderlines $\mathcal{M}_{o}$ that must be retrieved before a deadline $d_{o}$. Each orderline $m$ has two associated parameters: a weight $q_{m}$ and a dedicated pick location $l_{m} \in \mathcal{L}$ from where to retrieve it. Let $\mathcal{K}$ be a set of order pickers equipped with carts of capacity $W$. In each picker's tour $v$, a set of orderlines $\mathcal{M}_{v}$ have to be retrieved from their corresponding pick locations in a sequence visit $\mathcal{S}_{v}$. The processing time $p_{v}$ of a tour $v$ is defined as the sum of: a setup time $\beta^{s}$; a fixed search and pick 
time $\beta^{p}$ for each picked orderline; and a travel time that depends on the sequence visit $\mathcal{S}_{v}$. We aim at constructing a picking plan $\Pi=\left\{\Pi_{k}\right\}_{\{k \in \mathcal{K}\}}$, where $\Pi_{k}$ corresponds to a set of sequenced picking tours that must be performed by the picker $k$ during his/her work-shift such as: 1 . each order is completely picked before its deadline; 2 . the charge of each tour (cumulative weight of its orderlines) does not exceed the cart capacity; 3 . the tours assigned to each picker do not overlap; 4 . the total tours processing time is minimized.

\section{Route first-Schedule second heuristic}

We propose in this section a matheuristic to tackle the problem. Algorithm 1 describes the general structure of our approach. It is composed of two phases: routing phase (lines 1-5) and scheduling phase (line 6). The routing phase regroups the customer orders into clusters and constructs a set of picking tours for each cluster of orders. The clusters are built using the procedure constructClusters $(\mathcal{O})$. This procedure assembles greedily the orders that have close deadlines. To build the picking tours associated with each cluster, we ignore the time constraints to obtain a variant of the order batching problem in which we allow the orders splitting when we construct the picking tours (batches). This problem is solved using the procedure orderlinesBatching-SplitBasedProcedure $\left(\mathcal{O}^{c}\right)$. This latter starts by computing a giant tour that starts from depot, retrieves all orderlines of the current cluster of orders, and comes back to depot. It then uses an adaptation of the split algorithm to optimally extract a set $\Pi^{c}$ of tours that satisfy the capacity constraints from the giant tour. The tours generated for each cluster are added to the solution $\Pi$. After finding the tours of all clusters, the routing phase ends and the scheduling phase starts. In this phase, We formulate a constraint programming model that assigns the picking tours in $\Pi$ to the order pickers and schedules the tours assigned to each order picker such as the tours deadlines are satisfied.

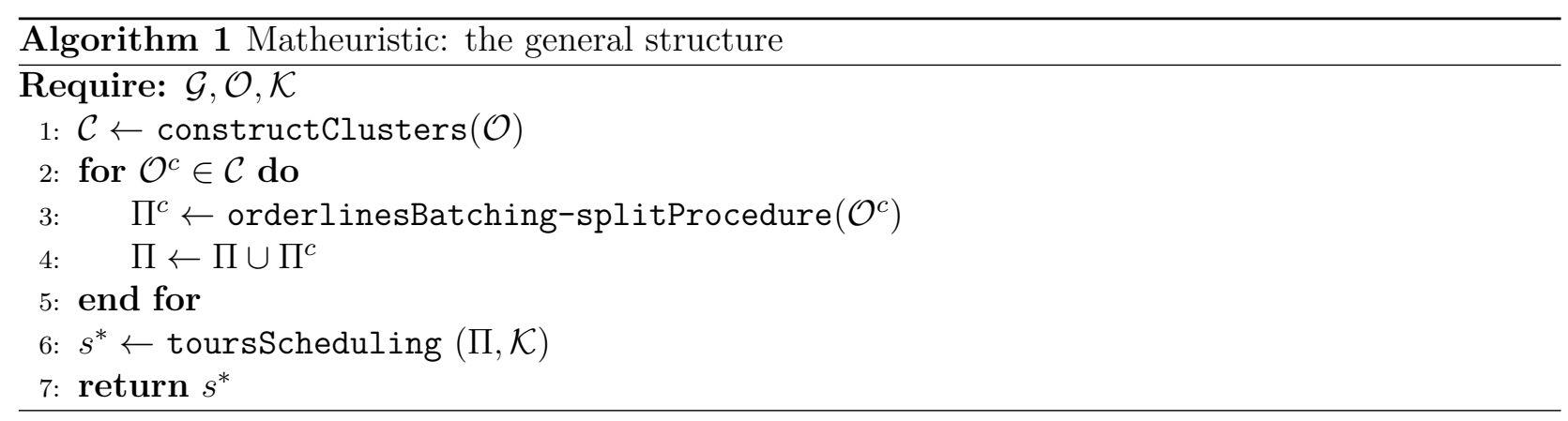

\section{Computational experiments}

We tested our heuristic (RFSS) over a data set of 2970 instances with different orders characteristics and different warehouse environments generated by [1]. We compared the total order picking time of the solutions delivered by our heuristic with that of the solutions retrieved by the ILS of [1]. We found that by allowing order splitting, our heuristic delivers a reduction of $30 \%$ on average and $60 \%$ in the best case. For a planning horizon of $4 h$, it represents more than a $1 h$ reduction of the shift of each picker on average with a maximum reduction of $1.8 \mathrm{~h}$.

\section{References}

[1] Van Gils, Teun and Caris, An and Ramaekers, Katrien and Braekers, Kris, Formulating and solving the integrated batching, routing, and picker scheduling problem in a real-life spare parts warehouse, European Journal of Operational Research, vol., no.3, pp. 814-830, 2008.

[2] Boysen, Nils and Stephan, Konrad and Weidinger, Felix Manual order consolidation with put walls: the batched order bin sequencing problem, EURO Journal on Transportation and Logistics, vol., no.2, pp. 169-193, 2019. 